\title{
Low-cost, Battery-Powered, Efficient Wireless Intelligent Sensor (LEWIS2): Outdoors and Remote Sensing Applications
}

\author{
Marlon Agüero*, Ali Ozdagli and Fernando Moreu \\ Department of Civil, Construction and Environmental Engineering, University of New Mexico, Albuquerque, NM 87131
}

\begin{abstract}
Structural Health Monitoring (SHM) systems are used to monitor critical infrastructure, such as bridges, high-rise buildings, and stadiums, and it has been observed that SHM has the potential to both prolong the structure lifespan and to improve public safety. Within SHM studies and in the research on monitoring in general, the application of wireless sensors (WS) has recently gained considerable research interest due to the cost reduction, associated with the installation and maintenance of monitoring systems. However, despite the advances in the research on WS, there are several issues and challenges in the application of WS that require improvements, in particular for Outdoor Environment Monitoring (OEM) because of their harsh operational conditions, huge targeted areas and limited energy budgets. This paper presents a low-cost, battery-powered, efficient wireless intelligent sensor (LEWIS2) that stores acceleration and angular velocity on a memory card to accurately estimate the trajectory of different applications.
\end{abstract}

\section{Introduction}

During the recent years, WS have been widely used in a variety of applications concerned with water monitoring [1-3], forest monitoring $[4,5]$, industrial monitoring [6, 7], aerospace monitoring, agriculture monitoring [8, 9], battlefield surveillance $[10,11]$, intelligent transportation $[12,13]$, smart homes [14, 15], animal behavior monitoring [16, 17], and disaster prevention $[18,19]$.

Outdoor environment monitoring (OEM) is of principal importance in civil engineering for the purpose of both protecting human life and avoiding economic loss due to structural disasters. The reliability of OEM systems needs to be further improved for two reasons. First, structures in urban areas are subject to unprecedented demand because of the growth in population and transportation, which gives rise to a higher level of deterioration of structures. Second, due to the process of climate change, buildings and infrastructure are more frequently affected by natural disasters, such as tropical storms and flooding, as witnessed during Hurricane Harvey in Houston in 2017 and earlier during Hurricane Katrina in New Orleans in 2005 [20-22]. In consequence, it is becoming increasingly important to develop systems of sensors which allow us to establish how structures behave under normal and extreme load conditions. Sensors are key devices for civil engineers, as they permit observations of infrastructure to an unprecedented level of detail. In a long run, these observations can be used by engineers to optimize system designs and improve maintenance operations. [23]

With the increased demand for sensor systems, the application of WS has recently gained considerable attention due to their cost reduction advantages and the possibility of performing high-density installation, which targets local damage and provides more detailed and reliable information about the dynamic properties of the structures. In consequence, new advances have been made in the development of WS to offer an economical method for collecting data that can be applied to make informed decisions about the dynamic performance of structures as well as the trajectory of displacements.

This paper presents the design of a low-cost, batterypowered efficient WS (LEWIS2), which can be used for outdoor applications to measure in real time angular velocity and acceleration, which are important parameters in many applications. In contrast to many traditional WS, which are technologically complex and costly, the proposed LEWIS2 system can be built with off-the-shelf components, at a cost of less than $5 \%$ of the average price of a commercial wireless sensor. More generally, this paper contributes to OEM capabilities for multiple field environments and structural responses of diverse infrastructures with low-cost, efficient wireless intelligent sensors.

\section{Low-cost, battery-powered efficient wireless intelligent sensor (LEWIS2)}

This section presents the structure of LEWIS2 and provides recommendations of specific components that could be used for LEWIS2 on the basis of their characteristics and availability on the market.

\subsection{Wireless sensor node elements}

\footnotetext{
* Corresponding author: magueroinjante $@$ unm.edu
} 
Generally, a WS node is made of the following essential parts: sensor, microcontroller, external memory, transceiver, and power source [24]. The sensor or sensing unit acquires data from the field and converts it into digital data. The microcontroller is used as a processor to acquire and process the sensor raw data to store the results to an external memory. The external memory may save a large amount of data, enabling posterior access by the user and remote sensing abilities. The transceiver unit enables sharing data between the base station or other wireless nodes. The final element is a power unit which consists of an energy source (capacitor, battery, or both of them). Depending on application requirements, further modules can be added. Figure 1 presents the way these elements are interconnected.

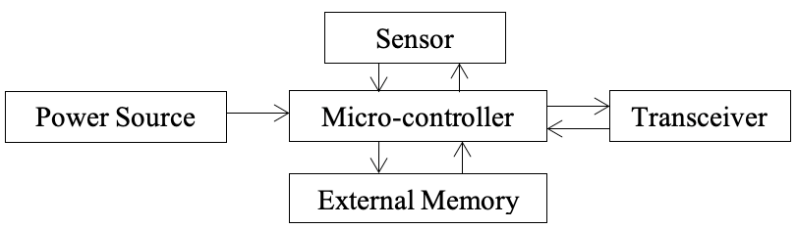

Fig. 1. Interconnection of basic components of a WS.

\subsection{Elements of the proposed LEWIS2}

This section outlines the key components of WS and provides recommendations of brands that the researchers used in the development of LEWIS2.

\subsubsection{Microcontroller}

Arduino Uno R3 is an open-source microcontroller board, based on the ATmega328P microcontroller. The board contains the following components: 14 digital input/output pins (which is used to read information from the sensor and to control the actuator, respectively), six of which can be applied as PWM outputs), 6 analog inputs, a USB connection, a power jack, a $16 \mathrm{MHz}$ quartz crystal, an ICSP header, and a reset button [25]. It can be powered from AC adapters, most USB chargers, or the USB port through a computer. Arduino Uno R3 has numerous advantages. It is flexible, features a userfriendly interface, and enables smooth communication between devices [26].

\subsubsection{Sensor}

LEWIS2 employs a multi-chip module (MCM) MPU9250, produced by TDK InvenSense. The MPU9250 is a multi-chip module that comprises two dies that are integrated into one QFN (Quad-Flat Noleads) package. The sensor has a wide dynamic measurement range and high output resolution of 14 bit $(0.6 \mu \mathrm{T} / \mathrm{LSB})$ or 16 bit $(15 \mu \mathrm{T} / \mathrm{LSB})$. It is equipped with an accelerometer with a dynamically selectable measurement range that varies from $\pm 2 \mathrm{~g}$ to $\pm 16 \mathrm{~g}$ in all the three axes. The Arduino Uno uses the I2C interface to accesses acceleration data sampled by the MPU9250. A breakout board produced by Adafruit Industries contains the sensor unit. The board has been connected to the Arduino board through a breadboard with minimal soldering and wiring. To configure the settings of the sensor, the researchers made use of an open-source Arduino library.

\subsubsection{Transceiver}

\subsubsection{Arduino wireless SD shield}

Arduino Wireless SD Shield, produced by Arduino, is a device that performs two functions. First, it permits the Arduino board to perform wireless communication via a wireless module, in particular, the XBee transceiver module, but it can be also used with other modules that have the same footprint to form mesh networks. Second, it features a micro SD card socket, which permits storing digital sensor data. It also contains a perfboard on the shield that can be used for prototyping circuits [25]. The micro SD socket included in the Arduino Wireless SD shield can be interfaced with the Arduino SD library. The two functions of the shield (storing and accessing data and interfacing with XBee transceiver modules) can be utilized independently and together.

\subsubsection{XBee series 1 module}

XBee Series 1 Module is a very simple and reliable device that permits communication between computers, systems, and microcontrollers. According to the Digi XBee documentation [27], the module has the range of 100 feet indoors and 300 feet outdoors with line-of-sight. It is very easy to set up and no prior configuration is required to operate it in peer to peer environments, which means that it can replace wired serial connections that were formerly used. The module is equipped with a wire antenna of excellent quality. The researchers selected the XBee module because it enables reliable wireless connection between LEWIS2 and other devices.

\subsubsection{Explorer}

XBee Explorer is an USB to serial base unit that can be applied in the Digi XBee line. The unit is very simple to use and is compatible with all the XBee modules, which include the standard and Pro versions of the Series 1 and Series 2.5. To operate an XBee, it must be plugged into the XBee Explorer, and connected via a mini USB-cable to a computer of a cellphone. This gives direct access to the serial and programming pins located on the XBee unit [28].

\subsubsection{Power source}

Nanotech Battery was used as the power source. The most important specifications of the battery are listed in Table 1 [29].

Table 1. Specifications of Nanotech 1.0 Battery.

\begin{tabular}{|c|c|}
\hline Capacity & $1000 \mathrm{mAh}$ \\
\hline Voltage & $2 \mathrm{~S} 1 \mathrm{P} / 2 \mathrm{Cell} / 7.4 \mathrm{~V}$ \\
\hline Weight & $60 \mathrm{~g}$ (including wire, plug and case) \\
\hline Dimensions & $71 \times 35 \times 12 \mathrm{~mm}$ \\
\hline
\end{tabular}


These properties make it a reliable battery that can be used to provide energy to the wireless sensor, so that it can work in an autonomous way. Importantly, the battery is rechargeable, so it can be charged with a solar panel. The elements of the proposed LEWIS2 are given in Figure 2 and the assembled LEWIS2 is illustrated in Figure 3. Additionally, Table 2 shows a cost summary of the elements of LEWIS2, with a description of each element.

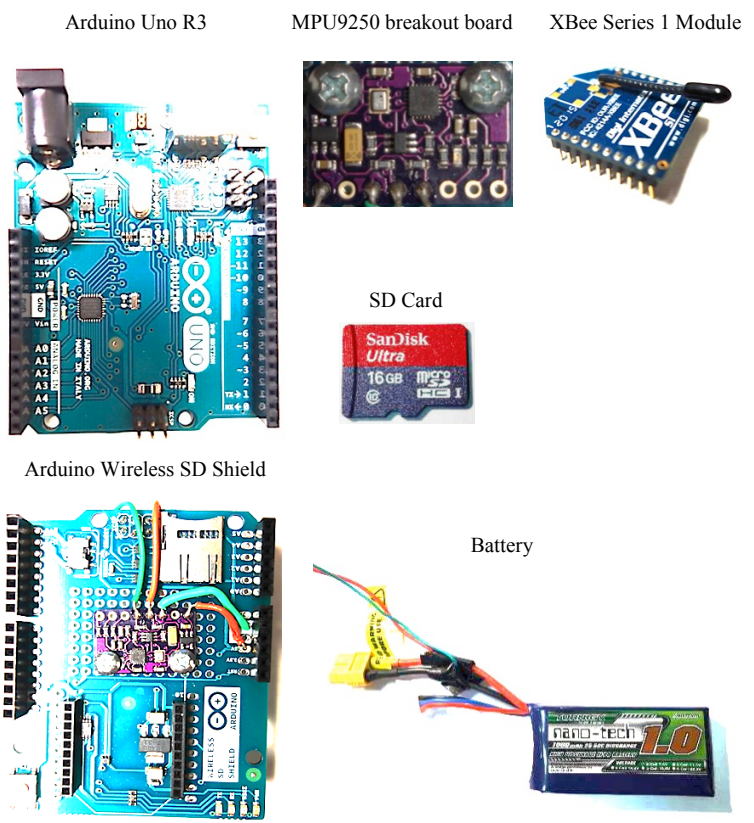

Fig. 2. Components of the Arduino-based LEWIS2.

\subsubsection{External memory}

The SD card uses Arduino Wireless SD shield. The communication between the microcontroller and the SD card uses SPI (Serial Peripheral Interface). The researchers used the Arduino SdFat library, which supports FAT16 and FAT32 file systems on both standard and high capacity SD cards, and which secures read/write access to FAT16/FAT32 file systems on $\mathrm{SD} / \mathrm{SDHC}$ flash cards. The researchers chose not to use the Arduino generic SD library because it is relatively slow.

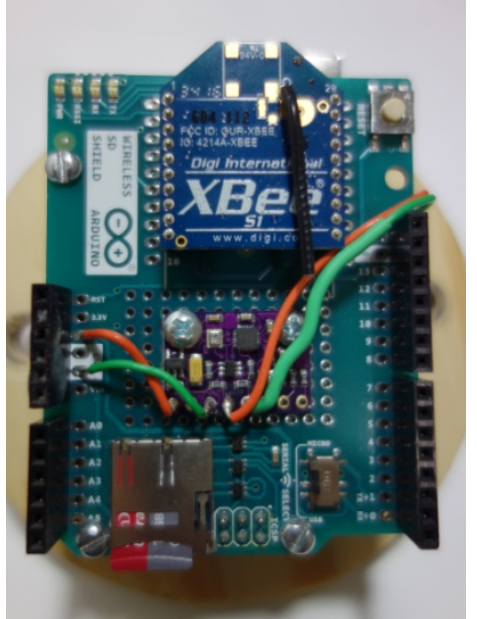

Fig. 3. Assembled LEWIS2.

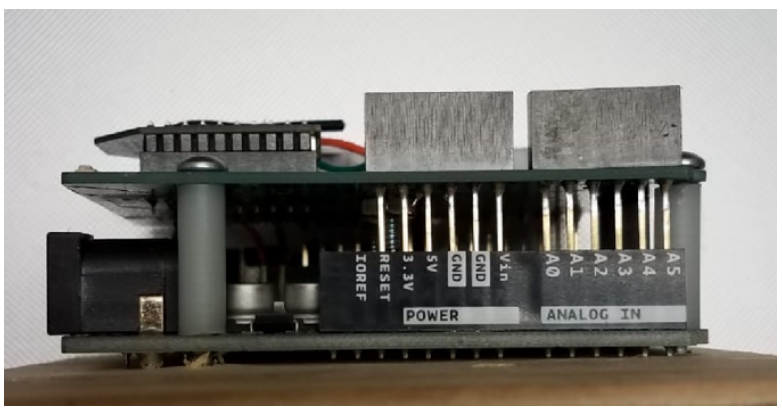

Fig. 4. Assembled LEWIS2 - side view.

Table 2. Essential sensing platform components LEWIS2.

\begin{tabular}{|c|c|c|}
\hline Element & Description & Price, $\$$ \\
\hline $\begin{array}{c}\text { Arduino Uno } \\
\text { R3 }\end{array}$ & Microcontroller & $\$ 4.00-\$ 25.00$ \\
\hline MPU9250 & Sensor & $\$ 9.99$ \\
\hline $\begin{array}{c}\text { XBee Series 1 } \\
\text { Module }\end{array}$ & $\begin{array}{c}\text { Wireless transmission } \\
\text { module }\end{array}$ & $\$ 25.00$ \\
\hline $\begin{array}{c}\text { Arduino } \\
\text { Wireless SD } \\
\text { Shield }\end{array}$ & $\begin{array}{c}\text { Communication XBee S1 } \\
\text { Module and Arduino } \\
\text { Uno R3 }\end{array}$ & $\$ 15.00$ \\
\hline Battery & $\begin{array}{c}\text { Nanotech LiPo 1000mAh } \\
\text { rechargeable battery }\end{array}$ & $\$ 6.33$ \\
\hline SD Card & $\begin{array}{c}\text { SanDisk Ultra 80MBs } \\
\text { MicroSD Memory Card - } \\
\text { 16GB }\end{array}$ & $\$ 10.00$ \\
\hline & Total & $\mathbf{\$ 7 0 . 3 5 - \$ 9 1 . 3 5}$ \\
\hline
\end{tabular}

The authors of this paper verified the workings of the LEWIS2 sensor by placing it on the tower of the Sandia Peak Aerial Tramway, near Albuquerque, NM, to register its vibrations under traffic operations. With the range for acceleration measurement of $+/-16 \mathrm{~g}$ and for the angular velocity +/- 2000 degree per second, LEWIS2 was able to record the full acceleration and angular velocity profile of the tramway's motion.

\section{Trajectory estimation with LEWIS2}

LEWIS2 can provide information about acceleration and angular velocity. On the basis of these data it is also possible to draw informed conclusions about trajectory. 
In line with Zhi's [30] procedure, the authors of this paper used an algorithm, as shown in Figure 5 with the steps listed below in order to obtain trajectory while applying LEWIS2.

Step 1: We pass the data of accelerometer and gyroscope to an explicit complementary filter (ECF). The filter provides us with output quaternions for the complete series of data. The attitude that we wish to obtain can be represented by quaternions or by their transformation into some other rotation representation. When the Matlab code is applied, the quaternions become transformed into rotation matrix and produce 3D-trajectory monitor animations.

Step 2: We apply the quaternion that was computed in step 1 in order to have the accelerometer data rotated from the sensor frame to the Earth frame.

Step 3: We integrate the acceleration in Earth frame received in step 2 in order to obtain velocity in the earth frame.

Step 4: The accelerometer data is passed to the Zero Velocity Update (ZUPT) filters (which include a firstorder Butterworth low-pass filter and a first-order Butterworth high-pass filter) in order to obtain the processed magnitude of acceleration and to determine stationary periods.

Step 5: The stationary periods are applied to zeroupdate the velocity that was computed in step 3 in order to obtain ZUPT velocity in the Earth frame.

Step 6: The drift occurring during the non-stationary periods is removed because of integration.

Step 7: We perform integral on linear velocity that was computed in step 6 in order to obtain the position in the Earth frame.

Step 8: The position data is passed to Enhanced Heuristic Drift Elimination (EHDE) in order to obtain the estimation of the drift eliminated position in the Earth frame.

Once these steps are followed, attitude estimation is obtained from step 1, whereas drift eliminated displacement is rendered by step 8 (see Zhi [30]).

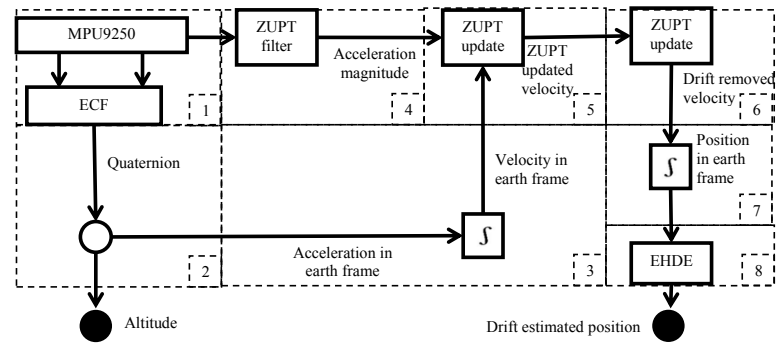

Fig. 5. Steps to obtain trajectory with LEWIS2.

Significantly, drift reduction techniques are applied within each integral level. Thus, in the level of acceleration, gyro drift becomes reduced through the implementation of ECF with a PI controller. In the level of velocity, integral drift of acceleration is reduced thanks to the implementation of ZUPT. In the level of displacement, the drift triggered by small errors coming from two lower levels which became integrated into a larger drift becomes removed through the implementation of the EHDE.

\section{Conclusions}

This paper has introduced a low-cost, battery-powered, efficient wireless intelligent sensor called LEWIS2. It is equipped with an SD card and a Nanotech 1.0 Battery. The new LEWIS2 platform can collect data with lowcost sensor, MPU9250, measuring acceleration and angular velocity of different outdoor applications.

LEWIS2 can work in environments with high vibration and displacements. It is capable of measuring acceleration and angular velocity to accurately estimate trajectories.

The financial support of this research is provided in part by the Department of Civil Engineering at the University of New Mexico, the Center for Teaching and Learning of the University of New Mexico under Teaching Allocation Grant, the New Mexico Consortium under grant number 249-01, and the Transportation Consortium of South-Central States (TRAN-SET), US Department of Transportation (USDOT) Project No. 17STUNM02. The authors of this paper thank the Canadian National Railway $(\mathrm{CN})$ for the data collected on the field to inform this proposed method. The conclusions of this research solely represent those of the authors.

\section{References}

1. Lee, H., Banerjee, A., Fang, Y., Lee, B., and King, C. (2010). Design of a multifunctional wireless sensor for in situ monitoring of debris flows. IEEE Trans. Instrum. Meas. 59, 2958-2967.

2. Perez, C., Jimenez, M., Soto, F., Torres, R., López, J., and Iborra, A. (2011). A system for monitoring marine environments based on Wireless Sensor Networks. Santander, Spain: In Proceedings of the IEEE Conference on OCEANS pp. 1-6.

3. Jiang, P., Xia, H., He, Z., and Wang, Z. (2009). Design of a Water Environment Monitoring System Based on Wireless Sensor Networks. Sensors 2009, 9, 6411-6434.

4. Bayo, A., Antolín, D., Medrano, N., Calvo, B., and Celma, S. (2010). Early Detection and Monitoring of Forest Fire with a Wireless Sensor Network System. Proced. Eng. 2010, 5, 248-251.

5. Yunus, E., Ibrahim, K., and Özgür, U. (2012). A framework for use of wireless sensor networks in forest fire detection and monitoring. Comput. Environ. Urban Syst. 2012, 36, 614-625.

6. Silva, I., Guedes, L., Portugal, P., and Vasques, F. (2012). Reliability and Availability Evaluation of Wireless Sensor Networks for Industrial Applications. Sensors 2012, 12, 806-838.

7. Zhao, G. (2011). Wireless Sensor Networks for Industrial Process Monitoring and Control: A Survey. Netw. Protoc. Algorithms 2011, 3, 46-63.

8. Raul, M., Samuel, G., Miguel, A., António, L., Salviano, F., Ferreira, P., and Reis, M. (2008). Sun, wind and water flow as energy supply for small stationary data acquisition platforms. Comput. Electron. Agric. 2008, 64, 120-132.

9. Li, X., Deng, Y., and Ding, L. (2008). Study on precision agriculture monitoring framework based 
on wsn. In Proceedings of the 2nd International Conference on Anti-counterfeiting, Security and Identification (ASID 2008). Guiyang, China. 182185.

10. Qian, H., Sun, P., and Rong, Y. (2012). Design Proposal of Self-Powered WSN Node for Battle Field Surveillance. Energy Proced. 2012, 16, 753757.

11. Padmavathi, G., Shanmugapriya, D., and Kalaivani, M. (2010). A Study on Vehicle Detection and Tracking Using Wireless Sensor Networks. Wirel. Sens. Netw. Sensors 2014, 14, 16950.

12. Tacconi, D., Miorandi, D., Carreras, I., Chiti, F., and Fantacci, R. (2010). Using wireless sensor networks to support intelligent transportation systems. Ad Hoc Netw. 2010, 8, 462-473.

13. Tubaishat, M., Zhuang, P., Qi, Q., and Shang, Y. (2009). Wireless sensor networks in intelligent transportation systems. Wirel. Commun. Mob. Comput. 2009, 9, 287-302.

14. Lee, H., Wu, C., and Aghajan, H. (2011). Visionbased user-centric light control for smart environments. Pervasive Mob. Comput. 2011, 7, 223-240.

15. Bangali, J., and Shaligram, A. (2013). Energy efficient Smart home based on Wireless Sensor Network using LabVIEW. Am. J. Eng. Res. 2013, 2, 409-413.

16. Handcock, R., Swain, D., Bishop-Hurley, G., Patison, K., Wark, T., and Valencia, P. (2009). Monitoring Animal Behaviour and Environmental Interactions Using Wireless Sensor Networks, GPS Collars and Satellite Remote Sensing. Sensors 2009, 9, 3586-3603.

17. Nadimi, E., Jørgensen, R. B.-V., and Christensen, S. (2012). Monitoring and classifying animal behavior using ZigBee-based mobile ad hoc wireless sensor networks and artificial neural networks. Comput. Electron. Agric. 2012, 82, 44-54.

18. Bahrepour, M., Meratnia, N., Poel, M., Taghikhaki, Z., and Havinga, P. (2010). Distributed event detection in wireless sensor networks for disaster management. Thessaloniki, Greece: In Proceedings of the 2010 2nd International Conference on Intelligent Networking and Collaborative Systems (INCOS), 507-512.

19. Lacono, M., Romano, E., and Marrone, S. (2010). Adaptive monitoring of marine disasters with intelligent mobile sensor networks. Taranto, Italy: In Proceedings of the 2010 IEEE Workshop on Environmental Energy and Structural Monitoring Systems (EESMS), 38-45.

20. Eamon, C.D., Fitzpatrick, P., and Truax, D. D. (2007). Observations of Structural Damage Caused by Hurricane Katrina on the Mississippi Gulf Coast. Journal of Performance of Constructed Facilities 21(2), 117-127.

21. Padgett, J., DesRoches, R., Nielson, B., Yashinsky, M., Kwon, O.-S., Burdette, N., and Tavera, E. (2008). Bridge Damage and Repair Costs from Hurricane Katrina. Journal of Bridge Engineering 13(1), 6-14.
22. Chen, Q., Wang, L., and Zhao, H. (2009). Hydrodynamic Investigation of Coastal Bridge Collapse during Hurricane Katrina. Journal of Hydraulic Engineering 135(3), 175-186.

23. Spencer, J.B., Sandoval, M.E., and Kurata, N. (2004). Smart Sensing Technology: Opportunities and Challenges. Journal of Structural Control and Health Monitoring 11(4), 349-368.

24. Akyildiz, I., Su, W., Sankarasubramaniam, Y., and Cayirci, E. (2002). Wireless sensor networks: a survey. Else Comput Netw 2002; 38, 393-422.

25. Arduino. (2018). Arduino Uno Rev3. $<$ https://www.arduino.cc/en/Guide/Environment $>$ (July 26, 2018).

26. Banzi, M., and Shiloh, M. (2015). Make: Getting Started with Arduino. Third edition. Sebastopol, CA: Maker Media.

27. XBee, D. (2018). Digi XBee ${ }^{\circledR}$ Family Features Comparison. Retrieved from https://www.digi.com/pdf/chart_xbee_rf_features.pd f (July 28, 2018).

28. XBee Explorer. (2018). SparkFun XBee Explorer USB. Product information retrieved from https://www.sparkfun.com/products/11812.

29. Nanotech. (2018). Turnigy nano-tech 1000mah $2 \mathrm{~S}$ 25 50C Lipo Pack. Product information retrieved from https://hobbyking.com/en_us/turnigy-nanotech-1000mah-2s-25-50c-lipopack.html? store $=$ en_us .

30. Zhi, R. (2016). A Drift Eliminated Attitude \& Position Estimation Algorithm In 3D. Graduate College Dissertations and Theses. Paper 450, University of Vermont. 